\author{
Proceedings of the $10^{\text {th }}$ International Conference on Applied Informatics \\ Eger, Hungary, January 30-February 1, 2017. pp. 5-13 \\ doi: 10.14794/ICAI.10.2017.5
}

\title{
FIR Filter Design Based Neural Network
}

\author{
Dhulfiqar A Alwahab ${ }^{a}$, Dhafer R. Zaghar ${ }^{b}$, Sándor Laki $^{c}$
}

\author{
${ }^{a}$ Eötvös Loránd University, Faculty of Informatics \\ aalwahab@inf.elte.hu \\ ${ }^{b}$ AL-Mustansytia University \\ drz_raw@yahoo.com
}

${ }^{c}$ Eötvös Loránd University, Faculty of Informatics

lakis@elte.hu

\begin{abstract}
The Finite Impulse Response (FIR) filter is one of the most important components in digital communications. Therefore, any enhancement of the FIR filter design will improve the efficiency of digital communications. There are several methods proposed to design or improve FIR filters. Involving Artificial Neural Network (ANN) in the FIR designing process is a modern technique that benefits from the high flexibility and non-linearity properties of the ANN. Many works have been proposed in this field, but most of them use random initial values to train neural networks, often producing results with unpredictable quality (weak and not optimal). On the other hand, other researches propose methods for modifying the transfer function of the FIR filter with the aim of enhancing the method, also leading to unsatisfied results in general cases. In this paper, we propose a novel solution to overcome the limitations of previous works by applying a modified training methods. The proposed method considers the ideal FIR filter as a target value, whereas using a pre-existing (window) methods to obtain the initial value and then use a modified back propagation algorithm (error value modification) to train the neural network. The modification in the error value gives the ability for increasing the stop-band ripple or decreasing the pass-band ripple separately, depending on the desired FIR filter.
\end{abstract}

Keywords: FIR, Kaiser window, Firls window, Rectangular window, Remez exchange, ANN, Back propagation

\section{Introduction}

Digital filters are usually used to eliminate the additional noise in signal which can affect negatively on the required portion. In many cases as in telephone systems, 
there is no need to transmit very high frequencies since most speech falls within the band of 400 to $3400 \mathrm{~Hz}$ [1]. Filtering out or rejecting all the frequencies above and below the desired band is mandatory in most Digital Signal Processing (DSP) systems. Digital filters are falling in two types; Finite Impulse Response (FIR) and Infinite Impulse Response (IIR). Linearity and stability properties of FIR give the reason of using this type in most communication systems [2]. On the other hand, FIR filters have greater flexibility in controlling the shape of their phase and magnitude response when comparing with IIR filters[3]. Based on the form of frequency transfer function, the FIR digital filter classified to; low-pass, high-pass, band-pass and band-stop filter. Fig.1, shows the general representation of low-pass FIR filter, where the output of this filter is given by:

$$
y[n]=b_{0} x[n]+b_{1} x[n-1]+b_{2} x[n-2]+\ldots \ldots \ldots b_{n} x[N]
$$

where, $\mathrm{N}$ is the order of the filter, $\mathrm{x}[\mathrm{n}]$ is the input of the signal and $\mathrm{b}$ represent the coefficients of the FIR filter. Many methods were followed to design FIR filters like frequency sampling, Fourier analysis, and window methods. The most famous approach in FIR filter design, before Artificial Neural Networks (ANNs) was the window method [4]. Many different types of window had been used in the FIR implementation process such as Kaiser, Hamming, Hanning, rectangular, Firls, etc. The concept of the window method based on transforming an ideal duration of infinite impulse response to a finite duration impulse response filter design [5]. All the methods for FIR design that used in the DSP field are approximated approaches, so they don't give optimal results in the design of the FIR, therefore, the new researches proceed to use the ANN approach to solve this problem.

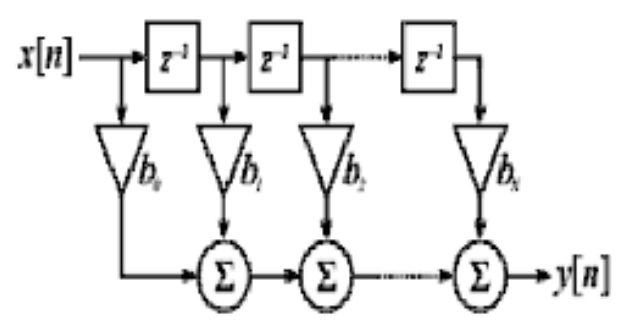

Figure 1: General representation of FIR filter[5]

\section{Related Works}

In [6], a low pass filter is implemented based on ANN, Self-Organizing Map (SOM) algorithm is used to train the network, the results show that ANN with SOM algorithm and Kaiser window method are almost same. In [2], Generalized Regression Neural Network (GRNN) is used to design FIR filter, this work has also been compared with the rectangular window method, the results give a very high accuracy 
when compared with Multi-layer Perceptron (MLP) back propagation algorithm. In [1], the authors conclude that ANN is the best and cheapest method for FIR filter design since in ANN, the coefficients of the filter can be calculated from unknown parameter in a specific range. In [5], a comparison between different types of ANN in FIR (low pass) filter design is investigated using FDA toolbox, in MATLAB, and Hamming window method. In [7], a comparison between back propagation and genetic algorithm were investigated, experiment result proves that ANN with back-propagation leads to better outcomes than genetic algorithm. In [8], FIR filter of order 10 is designed using MLP back propagation algorithm to train the ANN, the designed filter is compared with Hanning and triangular window, results illustrate that the designed filter by ANN and window method have almost the same output. [9], Sharma and Lahariya design and analyze a low pass FIR filter using different artificial neural network algorithm. In [10], FIR filter designed using ANN, then an optimization on the filter is implemented using genetic algorithm, after the optimization process the obtained method compared with Kaiser window, results show that optimization of FIR filter using ANN produces better results than Kaiser window in number of iterations.

It had been noticed that, the previous works have two common weak points. First, they start with random initial values in the neural network training process. Therefore, they reach to a different result (sometimes the results are weak or at least they are not optimal). Second, the previous works do not have the capability for enhancing only the stopband or passband. This work suggests a novel solution to overcome these two weak points as a step to enhance the FIR design using ANN.

\section{FIR Implementation Using ANN}

The FIR filter shown in Fig.1 could be modified to be a feed forward ANN in three main steps: First, convert the serial input data in FIR filter to parallel input using serial to parallel converter, (D), were D-flip flop used in this implementation. Second, consider the coefficients b in Eq. (1.1), as the weights w of ANN by noticed that ANN uses algorithms to calculate the weights and train the network such as genetic algorithm, GRNN, Back Propagation (BP). Finally, the third step, alter the output function in FIR filter which is a direct value of the sum to be a function of the summation exactly the same one used in ANN. Fig.3 shows the modification process on the FIR filter.

Among all the algorithm used in $\mathrm{ANN}, \mathrm{BP}$ is the simplest [5]. The $\mathrm{BP}$ use gradient descent approach to seek for the minimum of the error function in weight space. This approach solves the problem by combining the weights which minimize the error function. 


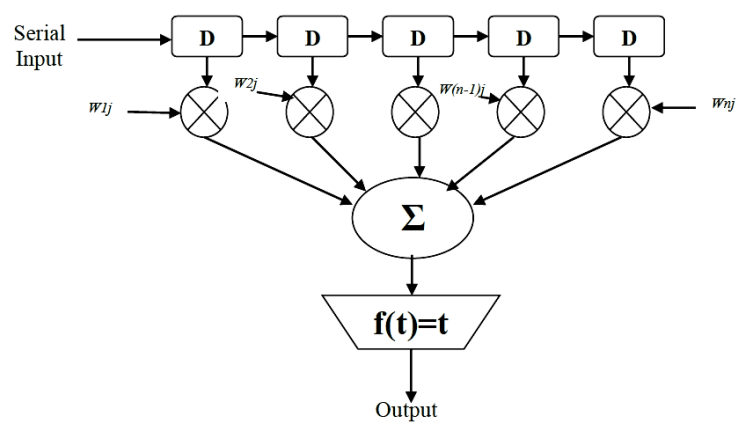

Figure 2: General representation of FIR filter using ANN

\section{Modified Neural Network Model}

Previous works trained the ANN through BP by following the classical (traditional) method for FIR filter design. This method for FIR filter design doesn't usually lead to a satisfy result. However, two novel issues will be proposed in this work with the aim of enhancing the filter response, which are the initial values for BP algorithm and the selected band of the desired FIR filter. These two issues have to be considered before training the Network.

\subsection{Initial Values}

BP starts with random weights and tries to modify these weights during the training process until achieving the best result (minimum error). But the weak point in BP is represented by the initial weights, since its beginning with random values, weight calculation (the learning process) will consume a long time and not reach to the global minimum (error) point, but it localizes in a local minimum point, so these values change with each learning cycle (run), the local minimum points will not give an optimal result for all times. Instead of starting with random weights, the values from window methods (classical method for FIR design) are proposed to be the initial values for BP algorithm, then use ANN to enhance these values. This method will produce an optimal result, but changing the initial random for BP by specific values has a negative effect on the ANN optimization process. Therefore, the rectangular window method has been adopted in this work because it usually provides the worst performance in FIR filter design. Additionally, some random values are also combined with the coefficient result of the rectangular window to be the initial values for $\mathrm{BP}$ to strength the ANN learning process. For instance, an FIR filter that had been designed in [11], with the following characteristics; a normalized LPF (0 to $\Pi \mathrm{Hz}$ ), order 51 , cutoff frequency 0.3 , transition range 0.05 , and passband ripple $3 \mathrm{~dB}$. This FIR filter has a stopband ripple $35 \mathrm{~dB}$ when designed using the rectangular window, $55 \mathrm{~dB}$ when designed using Firls window, while Remez exchange produces $62 \mathrm{~dB}$.The design of FIR filter using classical back 
propagation methods (with the same above characteristics) produces a filter with stopband ripple in the range of $(53 \mathrm{~dB}-68.4 \mathrm{~dB})$ for 100 runs, while using the proposed method for the initial values gives stopband ripple in the range of $(67.9$ $\mathrm{dB}-68.2 \mathrm{~dB}$ ) for the same number of runs. In result, the classical back propagation gives a higher stopband ripple $(53 \mathrm{~dB})$, while the proposed method enhanced the value of the stopband ripple in the range $(67.9 \mathrm{~dB}-68.2 \mathrm{~dB})$ at all times.

\subsection{ANN-based FIR Filter Implementation}

The neural networks based on algorithms to build models, these algorithms use data for training the network, poor or insufficient data in the training process will not produce an acceptable model. The previous works use the prefabricated software to find the transfer function in the frequency domain which means, learning process will enhance the passband and the stopband at the same time and in the same priority. Improving only the stopband or only the passband is not obtainable by these approaches. The proposed work suggests a different approach to calculate the transfer function in the frequency domain as in Fig.4. In this method, the output of the square wave generator can be represented by the following equation:

$$
f_{t}=\frac{1}{2} f_{c} \pm n \Delta f
$$

Where $f_{t}$ is the frequency of the square wave, $\mathrm{f}_{c}$ is the cutoff frequency of the FIR $\mathrm{LPF}, n$ integer number, and $\Delta \mathrm{f}$ is a very small value depends on the sharpening of the filter and it will limit to zero in the ideal FIR or at least it's smaller than half of the transition range.

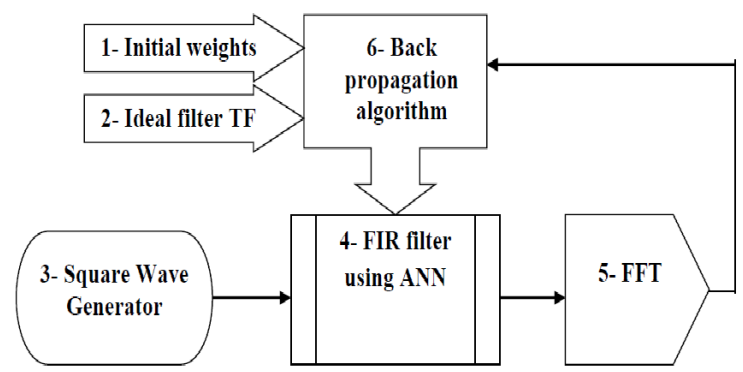

Figure 3: Complete circuit for the proposed Method

The design of the normalized FIR LPF under ANN in this work has 6 steps:

1. Calculate the initial values for the BP algorithm using classical FIR design method (rectangular window) and add some random values for the result of the design, the random value is obtained by Eq. (4.2) and (4.3):

$$
\begin{aligned}
\operatorname{Random}(j) & =0.1 * h_{\max } * \operatorname{random}(0,1] \\
w_{j} & =h_{j}+\operatorname{random}(j)
\end{aligned}
$$


where $h_{j}$ is the coefficients of the FIR filter that designed by the rectangular window and $h_{\max }$ is the maximum cofficient value.

2. Design the ideal filter that has a characteristic to meet the required system specifications, with stopband ripple, is $100 \mathrm{~dB}$ (because the ideal stopband ripple is $\infty \mathrm{dB})$.

3. Select the suitable frequency of the square wave generator as in Eq. (4.1). In this work, $f_{c}=0.3 \mathrm{~Hz}, \Delta f=0.02 \mathrm{~Hz}$, and $n=15$.

4. Calculate the outputs of the FIR using Eq. (1.1). In this work, $\mathrm{N}=51$.

5. Convert the FIR outputs to the frequency domain using FFT. In this work, 256 points have been assumed.

6. Use BP algorithm to change the FIR coefficients (ANN weights) then, calculate the absolute difference between the old and new coefficients and repeat steps 4 to 6 until reaching to the saturation case (for this work the summation of the absolute difference between the old and new values is less than 0.0001).

Based on the fact, back propagation relies on the value of error to train the neural network. The modified BP will insert additional factor in the pre-initial values to calculate the error functions as in Eq. (4.4).

$$
E=b_{p} \sum_{i=0}^{a} e_{i}+b_{t} \sum_{i=a+1}^{b} e_{i}+b_{s} \sum_{i=b+1}^{N-1} e_{i}
$$

where $\mathrm{E}$ is the total error, $e_{i}$ is the difference between instant and ideal values for the sample $\mathrm{i}, b_{p}, b_{t}$, and $b_{s}$, are the priority of passband, transition, and stopband respectively. In this work, $\mathrm{N}=(256)$, and $\mathrm{Pt}=1$. Eq. (4.4), gives the flexibility to enhance only one band ripple (stop or pass) at a time.

\section{Evaluation}

For the experiment purpose, three neural networks are obtained based on the modification in the error value. Neural Network 1 (NN1), this type has an error value in passband multiplied by $2\left(\mathrm{Pp}=2\right.$ and $\left.\mathrm{P}_{\mathrm{s}}=1\right)$. Neural Network $2(\mathrm{NN} 2)$, has a stopband error value multiplied by $2\left(\mathrm{Pp}=1\right.$ and $\left.\mathrm{Ps}_{\mathrm{s}}=2\right)$. Neural Network 3 (NN3), has no modification in any type of error $\left(P p=1\right.$ and $\left.P_{s}=1\right)$. Table $(1)$ shows the properties of LPF with order 51 when designed by the proposed neural networks versus some best well known traditional filter design methods. The first four methods (rectangular, Kaiser, Firls and Remez exchange) have a passband ripple $3 \mathrm{~dB}$, which is considered as high ripple, and transition range 0.05. Whereas, they have stopband ripple equal to $(35,52,55,62) \mathrm{dB}$ respectively. The ANN improves the stopband ripple to $68 \mathrm{~dB}$ in NN1 method. In NN2 The value of passband ripple 


\begin{tabular}{|l|l|l|l|l|}
\hline Method & Order & Pass ripple & Transition & Stop ripple \\
\hline Rectangular window & 51 & 3 & 0.05 & 35 \\
\hline Kaiser & 51 & 3 & 0.05 & 52 \\
\hline Firls window & 51 & 3 & 0.05 & 55 \\
\hline Remez exchange & 51 & 3 & 0.05 & 62 \\
\hline NN1 & 51 & 3 & 0.05 & 66 \\
\hline NN2 & 51 & $\mathbf{2 . 4}$ & 0.05 & $\mathbf{6 2}$ \\
\hline NN3 & 51 & $\mathbf{2 . 8}$ & 0.05 & $\mathbf{6 5}$ \\
\hline
\end{tabular}

Table 1: Comparison of classical methods of LPF design and NN approaches for high ripple passband.

is $2.4 \mathrm{~dB}$, and stopband ripple is $62 \mathrm{~dB}$. While the value of passband ripple and stopband ripple in $\mathrm{NN} 3$ are $2.8 \mathrm{~dB}$ and $65 \mathrm{~dB}$ respectively.

The same methods used in table (1) to design LPF with high ripple passband are also used to design LPF with a low ripple passband of the same order (51). Table (2) illustrates the variation in stopband ripple values, same transition range values to 0.05 and passband ripple values for all methods to 0.005 except NN1 method has (0.008). On the other hand, stop band ripple for these methods (rectangular, Kaiser, Firls, Remez, NN1, NN2 and NN3) are (23, 85, 39, 31, 88, 86 and 85) respectively.

\begin{tabular}{|l|l|l|l|l|}
\hline Method & Order & Pass ripple & Transition & Stop ripple \\
\hline Rectangular window & 51 & 0.005 & 0.05 & 23 \\
\hline Kaiser & 51 & 0.005 & 0.05 & 85 \\
\hline Firls window & 51 & 0.005 & 0.05 & 39 \\
\hline Remez exchange & 51 & 0.005 & 0.05 & 31 \\
\hline NN1 & 51 & $\mathbf{0 . 0 0 8}$ & 0.05 & $\mathbf{8 8}$ \\
\hline NN2 & 51 & $\mathbf{0 . 0 0 5}$ & 0.05 & $\mathbf{8 6}$ \\
\hline NN3 & 51 & $\mathbf{0 . 0 0 5}$ & 0.05 & $\mathbf{8 5}$ \\
\hline
\end{tabular}

Table 2: Comparison of classical methods of LPF design and NN approaches for low ripple passband

\section{Conclusion}

Traditional methods for FIR filter design such as windows or Remez exchange are approximate and do not usually produce an optimal result. The main problem of the ANN PB algorithm is random initial values which lead to unsatisfied and predicted results when used in FIR filter design. The proposed method is used to design a normalized LPF type FIR with order 51 as a practical example. It has been noticed from table $1 \& 2$ that, the classic methods are restricted by the design 
characteristics of the filter and not optimize or enhance the designed filter. As an example, Remez exchange gives a good result for high ripple (Table 1), but it fails in the low ripple (Table 2). While, the Kaiser window gives a good result for low ripple (Table 2), but it fails in the high ripple (Table 1). The ANN with random initial values sometimes gives not optimal results (stopband ripple is $53 \mathrm{~dB}-68.4$ $\mathrm{dB}$ as in the practical example), while the initial values proposed method gives an optimal result for all times (stopband ripple is $67.9 \mathrm{~dB}-68.2 \mathrm{~dB}$ for the same example). Hence, the best results without ANN is in Remez exchange that gives stopband ripple is $62 \mathrm{~dB}$ for the same example. The proposed method increases the flexibility of ANN by limiting the selecting band of the filter, since it gives the filter designer the opportunity to enhance only one band ripple (stopband or passband) at a time or both of them at the same time, depending on the required filter characteristic. This work enhances the FIR characteristics without any additional cost, results show that stopband ripple is raised in the range ( $3 \mathrm{~dB}$ to $4 \mathrm{~dB})$, and decrease the passband ripple to $(0.6 \mathrm{~dB})$ when compared with the best method used in FIR filter design. Other methods of ANN like Genetic Algorithm (GA), Practical Swarm Optimization (PSO), etc. with the same design steps that suggested in this work were planned for future work.

\section{References}

[1] Harpreet Kaur and Balwinder Dhaliwal Design of Low Pass FIR Filter Using Artificial Neural Network, International Journal of Information and Electronics Engineering vol. 3, no. 2 (2013), pp. 204-207

[2] Navneet Gupta and Ravindra Pratap Narwaria Design Low Pass FiR Filter Using Generalized Regression Neural Network, International Journal of Signal Processing, Image Processing and Pattern Recognition Vol.7, No.2 (2014), pp.75-84

[3] M. Kumar, R. Patel, R. Saxena and S. Kumar Design of band pass finite impulse response filter using various window method, international journal of engineering Research and application Vol. 3, no. 5 (2013), pp. 1057-1061

[4] Mahesh S. Chavan, R. A. Agarwala and M. D. Uplane Use of Kaiser window for ECG processing, Proceedings of the 5th WSEAS Int. Conf. on Signal Processing, Robotics and AutomationMadrid, Spain February 15-17 (2006), pp.285-289

[5] M. A. Singh and V. B. V. Thakare Artificial Neural Network Use for Design Low Pass FIR Filter a Comparison, International Journal of Electronics and Electrical Engineering IJEEE no. 3 (2015), pp. 216-219

[6] N. Gupta, R. P. Narwaria, C. Shen, W. Tong, P. V. V. Reddy, L. Rajamani And S. H. Mirhosseini Study of FIR Filter Designing By Using Self Organizing Map Neural Network, International Journal of Advanced Science and Technology , Vol.70 (2014), pp. 1-10

[7] Che, Zhen-Guo, Tzu-An Chiang and Zhen-Hua CheFeed-forward neural networks training: A comparison between genetic algorithm and back-propagation learning algorithm, International Journal of Innovative Computing, Information and Control (2011), pp. 5839-5850 
[8] Aparna Timari, Vandana Thakre, and Karuna Markam Design of Low Pass FIR Filter Using Artificial Neural Network, FIR Filter Design Using Artificial Neural Network International Journal of Computer \& Communication Engineering Research vol. 2, no. 3 (2014),

[9] Suruchi Sharma and Abhishek Lahariya Design and Analysis of FIR Filter using Artificial Neural Network, International Journal of Computer and Electronics Research vol. 4, no. 3 (2015)

[10] Sheenu Thapar, Parminde Kaur and Neha Aggarwal A Low Pass Fir Filter Design Using Genetic Algorithm Based Artificial Neural Network, International Journal of Computer Technology and Electronics Engineering vol. 2, no.4 (2012) Springer-Verlag Berlin and Heidelberg GmbH \& Co. KG (2004)

[11] Zaghar, Dhafer Design and Implementation of General Digital Down Converter Using Field Programmable Gate Array, PhD diss., University of Baghdad Iraq, 2002. (2002) 